Journal for ImmunoTherapy of Cancer

\title{
Efficacy and immune-related adverse event associations in avelumab- treated patients
}

\author{
Karen Kelly (D) , ${ }^{1}$ Juliane Manitz, ${ }^{2}$ Manish R Patel, ${ }^{3}$ Sandra P D'Angelo (D) , ${ }^{4,5}$ \\ Andrea B Apolo (D) , ${ }^{6}$ Arun Rajan (D) , ${ }^{7}$ Vijay Kasturi, ${ }^{8}$ Isabell Speit, ${ }^{9}$ Marcis Bajars, ${ }^{2}$ \\ John Warth, ${ }^{2}$ James L Gulley (1) ${ }^{6}$
}

To cite: Kelly K, Manitz J, Patel MR, et al. Efficacy and immune-related adverse event associations in avelumabtreated patients. Journal for ImmunoTherapy of Cancer 2020;8:e001427. doi:10.1136/ jitc-2020-001427

Accepted 22 October 2020
Check for updates

(C) Author(s) (or their employer(s)) 2020. Re-use permitted under CC BY-NC. No commercial re-use. See rights and permissions. Published by BMJ.

For numbered affiliations see end of article.

Correspondence to

Dr Karen Kelly;

karkelly@ucdavis.edu

\section{ABSTRACT}

Background Adverse events (AEs) of special interest that arise during treatment with immune checkpoint inhibitors, including immune-related AEs (irAEs), have been reported to be associated with improved clinical outcomes. We analyzed patients treated with avelumab from the JAVELIN Solid Tumor and Merkel 200 trials, examining the association between AEs and efficacy while adjusting for confounding factors such as treatment duration and event order.

Methods We analyzed efficacy and safety data from 1783 patients treated with the programmed death ligand 1 inhibitor avelumab who were enrolled in expansion cohorts of the JAVELIN Solid Tumor and Merkel 200 trials. To analyze the association between irAEs and efficacy with regard to survival, we used a time-dependent Cox model with time-varying indicators for irAEs, as well as multistate models that accounted for competing risks and time inhomogeneity.

Results 295 patients (16.5\%) experienced irAEs and 454 patients (25.5\%) experienced infusion-related reactions. There was a reduced risk of death in patients who experienced irAEs compared with those who did not (HR 0.71, 95\% Cl 0.59 to 0.85) using the time-dependent Cox model. The multistate model did not suggest that the occurrence of irAEs could predict response; however, it predicted a higher chance of irAEs occurring after a response. No association was observed between response and infusion-related reactions.

Conclusions Patients who experience irAEs showed improved survival. Although irAEs are not predictors for response to immune checkpoint inhibitors, increased vigilance for irAEs is needed after treatment with avelumab.

Trial registration numbers NCT01772004 and NCT02155647.

\section{BACKGROUND}

Immune checkpoint inhibitors (ICIs) show a wide spectrum of clinical activity and are approved for use in a variety of solid tumors. ${ }^{12}$ However, ICIs are also associated with unique adverse events of special interest (AESIs), including immune-related AEs (irAEs) and infusion-related reactions (IRRs). ${ }^{3}{ }^{4} \mathrm{It}$ has been suggested that the occurrence of
irAEs and their corresponding management may compromise responsiveness to immunotherapy; however, there is conflicting evidence on the impact of immunosuppressive therapy used to manage irAEs on the clinical benefit of ICI therapy ${ }^{5-7}$ Patients who respond to treatment appear to have a higher likelihood of developing irAEs, and this might in part be related to increased immune cell activation, cross-reactivity between tumor neoantigens and normal tissue antigens or treatment duration ${ }^{8-10}$; however, not all autoimmune mechanisms elicited by ICIs are necessarily associated with antitumor response. ${ }^{11}$ Steroids are used for the management of irAEs, but accompanying immunosuppression can potentially compromise the antitumor response associated with immunotherapy; however, a short course of treatment with steroids generally does not have considerable impact. ${ }^{6} 8$ Immunotherapy dose interruption for management of irAEs can also compromise clinical activity. ${ }^{12}$

The development of irAEs has been associated with improved overall survival (OS) and clinical efficacy in various cancers. ${ }^{13-19}$ The mechanisms accounting for the association of irAEs with improved efficacy are still unknown, although shared antigens between normal and cancer cells may be one potential explanation ${ }^{10} 152021$; longer treatment duration is also a possible confounding factor for the development of irAEs. ${ }^{10}$ AEs are also linked to improved outcomes in other forms of cancer therapy, such as chemotherapy and kinase inhibitors. ${ }^{22-25}$

To date, comprehensive analyses of safety and efficacy that assess the potential association between the occurrence of response and AESIs, while adjusting for confounding factors, are either lacking or are not adequately performed. No appropriately powered studies exist that examine the association between IRRs and 
efficacy, adequately adjust for immortal time bias, as well as factor in treatment duration and/or the range of possible flexible event sequences. Studies also contradict each other in regard to whether an association exists. ${ }^{15} 16$ 26-29 Lastly, many analyses have sample sizes that were too small to sufficiently draw strong conclusions. ${ }^{29}{ }^{30}$ Thus, a proper understanding of the association between efficacy and safety using a multistate model that appropriately accounts for time dependency, treatment duration, and event order may improve the assessment of benefit/risk and aid in informed treatment decisions for patients with cancer.

Avelumab is a human anti-programmed death ligand 1 (PD-L1) immunoglobulin G1 monoclonal antibody that inhibits the interaction between PD-L1 and programmed cell death protein 1 (PD-1). ${ }^{31}$ Avelumab differs from other currently licensed IgG1 isotype anti-PD-L1 antibodies, namely atezolizumab and durvalumab, as the Fc receptors of the latter have been genetically modified to reduce their potential of binding to the FcyR1 receptor, thereby reducing or eliminating innate immune inducer functions. ${ }^{32}$ It has been shown in preclinical studies that avelumab induces innate effector functions against tumor cells in vitro. ${ }^{33}$ Avelumab is approved as monotherapy for metastatic Merkel cell carcinoma (MCC) and platinum-treated urothelial carcinoma (UC) in various countries, and in combination with axitinib for advanced renal cell carcinoma in the USA and European Union. We report findings on the association of efficacy and AESIs that were observed in patients who received avelumab in the JAVELIN Solid Tumor and JAVELIN Merkel 200 clinical trials.

\section{METHODS}

We pooled and analyzed efficacy and safety data from 1783 patients enrolled in expansion cohorts of JAVELIN Solid Tumor $(\mathrm{n}=1695)$ and part A of JAVELIN Merkel $200(\mathrm{n}=88)$. These trial designs have been described previously. $^{34}$ JAVELIN Solid Tumor (NCT01772004) is an international, multicohort, open-label, doseescalation and dose-expansion phase 1 trial of avelumab in patients with advanced solid tumors; JAVELIN Merkel 200 (NCT02155647) is an international, prospective, open-label, single-arm phase 2 trial of avelumab in patients who experienced disease progression after $\geq 1$ prior line of chemotherapy for metastatic MCC (part A). For both trials, additional patient eligibility criteria included immune-competent status; Eastern Cooperative Oncology Group performance status of 0 or 1; adequate hematological, hepatic and renal function; estimated life expectancy of $\geq 3$ months, $\geq 1$ unidimensional measurable lesion according to Response Evaluation Criteria in Solid Tumors (RECIST) version 1.1 (including skin lesions); and availability of fresh or archival biopsy material. Patients were not involved in the design or conduct of this study. All patients provided written informed consent.

Avelumab was administered at $10 \mathrm{mg} / \mathrm{kg}$ over a 1 -hour intravenous infusion every 2 weeks. AEs were assessed and graded by the National Cancer Institute Common
Terminology Criteria for Adverse Events V.4.0. The statistical analyses of AEs included treatment-emergent AEs only (ie, those with onset from start of study treatment up to 30 days after last study treatment). All serious AEs suspected to be related to the study treatment had to be reported regardless of the time elapsed since last treatment administration. irAEs were identified using a prespecified list of Medical Dictionary for Regulatory Activities preferred terms and then followed by a medical review of qualified medical personnel to determine whether the AE met all the criteria for an immune-related adverse reaction (onset, duration, immunosuppressive therapy, etiology). The association of efficacy and irAEs was analyzed using approaches that account for time dependency and treatment duration. A time-dependent Cox model considering time-varying indicators for irAEs was used for the analysis of OS. Furthermore, multistate models explicitly modeling competing risks and time inhomogeneity, while allowing different event order of response and irAE occurrence, were used to gain insights into underlying mechanisms. The multistate model considers a stochastic process representing the individual progress of each patient through different states while receiving avelumab treatment (figure 1) ${ }^{34}$ Each patient starts treatment at time zero, and treatment discontinuation is an absorbing state and includes discontinuation due to withdrawal, loss to follow-up, progressive disease, death or AE. As long as the patient is receiving treatment, experiencing an irAE, having a response or experiencing a combination state (irAE and response, in either order) are transient events. Occurrence of response and irAEs are modeled as transitions between the states of the model. Given that a patient is in state $i$ at time $t$, the probability that the patient is in state $j$ at time $s$ is given by:

$$
P_{i j}(s \mid t)=P(X(s)=j \mid X(t)=i), s>t
$$

Transition probabilities are time dependent (ie, the process is time inhomogeneous) and assume the Markov property (ie, a future state only depends on the present state and not on the history). All statistical approaches were used in an exploratory way and were performed in $\mathrm{R}^{35-37}$

IRRs (IRR, drug hypersensitivity or hypersensitivity) occurring on the day of or day after infusion and IRR symptoms occurring on the day of infusion that resolved within 2 days after onset were included in the list of prespecified AEs for analysis. Tumors were investigator assessed according to RECIST 1.1 every 6 weeks for the first 12 months after the start of study treatment and then every 12 weeks thereafter. Analyses of the association between achieving a best overall response (BOR) of confirmed complete response (CR) or partial response (PR) and IRRs were performed using two-way frequency tables, $\chi^{2}$ tests with Yates correction, boxplot analyses of time to response by occurrence of IRRs and Kaplan-Meier analysis of duration of response (DOR) grouped by the occurrence of IRRs. Adjustment for time dependency was not required because IRRs occurred early during treatment. 


\section{Questions to be addressed by multistate model:}

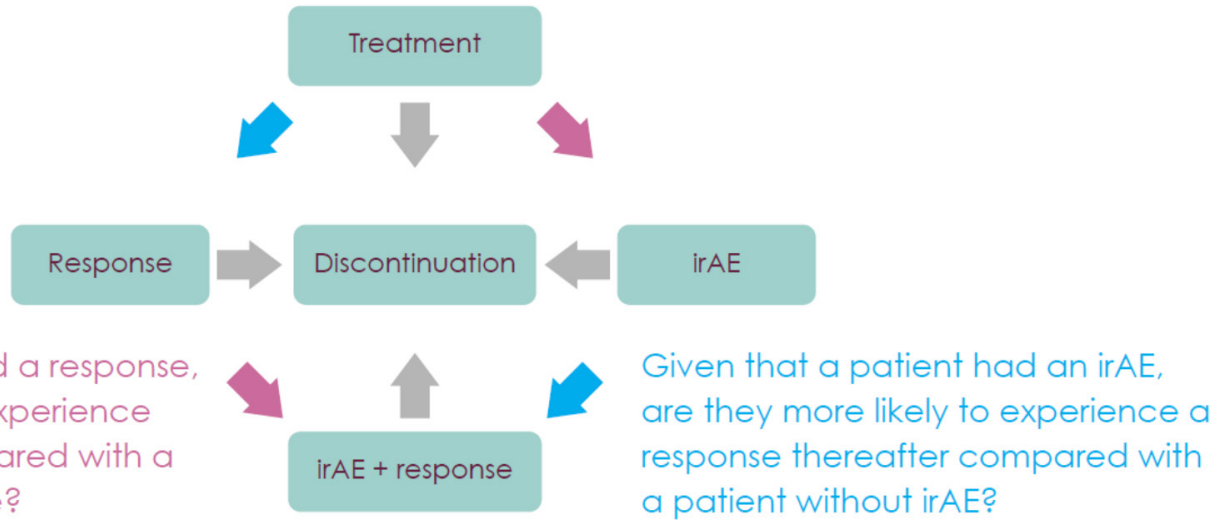

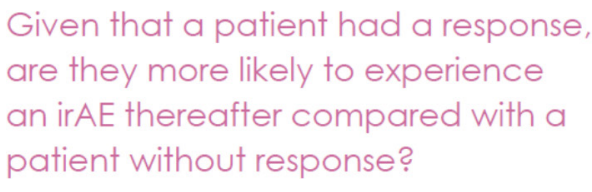

Given that a patient had a response. are they more likely to experi patient without response?

Figure 1 Multistate model definition and hypotheses to be addressed. Diagram shows the transition states that the multistate model aims to capture, which all begin with treatment, lead to either a response or irAE, and then may further lead to both response and irAE. Blue and pink arrows along with accompanying text describe two research questions regarding treatment sequencing to be investigated by the multistate model. irAE, immune-related adverse events.

\section{RESULTS}

The data cut-off dates for the JAVELIN Solid Tumor cohorts and part A of JAVELIN Merkel 200 were February 15 2017, and March 24 2017, respectively. The pooled data set contained 1783 patients with varying tumor types who were enrolled between September 2013 and October 2016 (table 1); all patients received $\geq 1$ dose of avelumab. Median duration of treatment was 12.0 weeks (range 2.0-172.9 weeks). The overall incidence of AESIs and

\begin{tabular}{|c|c|}
\hline Characteristics & $\mathrm{N}=1783$ \\
\hline Median age (range), years & $64(19-91)$ \\
\hline \multicolumn{2}{|l|}{ Sex, n (\%) } \\
\hline Male & $931(52.2)$ \\
\hline Female & $852(47.8)$ \\
\hline \multicolumn{2}{|l|}{ Geographic information, n (\%) } \\
\hline North America & $1267(71.1)$ \\
\hline Europe & 390 (21.9) \\
\hline Asia & $121(6.8)$ \\
\hline Australia & $5(0.3)$ \\
\hline \multicolumn{2}{|l|}{ Racial designation, n (\%) } \\
\hline White & $1343(75.3)$ \\
\hline Non-white & $436(24.5)$ \\
\hline No data & $4(0.2)$ \\
\hline \multicolumn{2}{|l|}{ ECOG PS, n (\%) } \\
\hline 0 & $680(38.1)$ \\
\hline$\geq 1^{*}$ & $1103(61.9)$ \\
\hline Mean prior therapies, $n$ (SD) & $2(0.87)$ \\
\hline
\end{tabular}

*Includes patients whose ECOG PS increased to $>1$ between screening and start of treatment.

ECOG PS, Eastern Cooperative Oncology Group performance status. onset of AESIs are shown in table 2 and figure 2. Patients with a BOR of $\mathrm{CR}$ or $\mathrm{PR}$ received treatment for a median of 14.3 months compared with 4.6 months for patients with stable disease (SD) and 1.4 months for patients with progressive disease or who were not evaluable.

In general, the incidence of AESIs by type were similar across tumor types (table 2). Of the 1783 patients in the study, 295 patients $(16.5 \%)$ experienced irAEs and 51 patients $(2.9 \%)$ experienced grade $\geq 3$ irAEs. Considering only the subset of patients who experienced irAEs $(\mathrm{n}=295), 57$ of these patients $(19.3 \%)$ first experienced a response and subsequently an irAE, 19 (6.4\%) first experienced an irAE before experiencing a response and 219 patients $(74.2 \%)$ did not have a response. Additionally, in this same subset, an irAE occurred in 87 patients $(29.5 \%)$ before the first tumor assessment (first to third infusion), in $87(29.5 \%)$ before the second tumor assessment (fourth to sixth infusion), and in 121 (40.8\%) later; thus, irAEs occurred throughout treatment. Grade $\geq 3$ irAEs were not observed in $>10 \%$ of the patients across tumor types; three deaths due to irAEs were observed. A 51-year-old female patient with metastatic breast cancer and known liver metastases experienced acute hepatic failure after administration of three infusions of avelumab. No autopsy was performed. Progression of underlying breast cancer metastatic to liver was considered as an alternative explanation for the event. A 50-year-old female patient with gastroesophageal junction cancer experienced grade 5 autoimmune hepatitis and grade 5 hepatic failure after 1 infusion of avelumab. Underlying cancer disease (gastric cancer/gastroesophageal junction cancer) and treatment with concomitant medications (lansoprazole, rebamipide, phazyme and oxycodone) was considered as an alternative explanation. A 54-year-old male patient experienced pneumonitis 19 days after the first and only infusion of avelumab. A CT scan revealed perihilar ground glass opacities (superimposed pneumonia could not be 
Table 2 Incidence and severity of IRRs and irAEs by tumor type

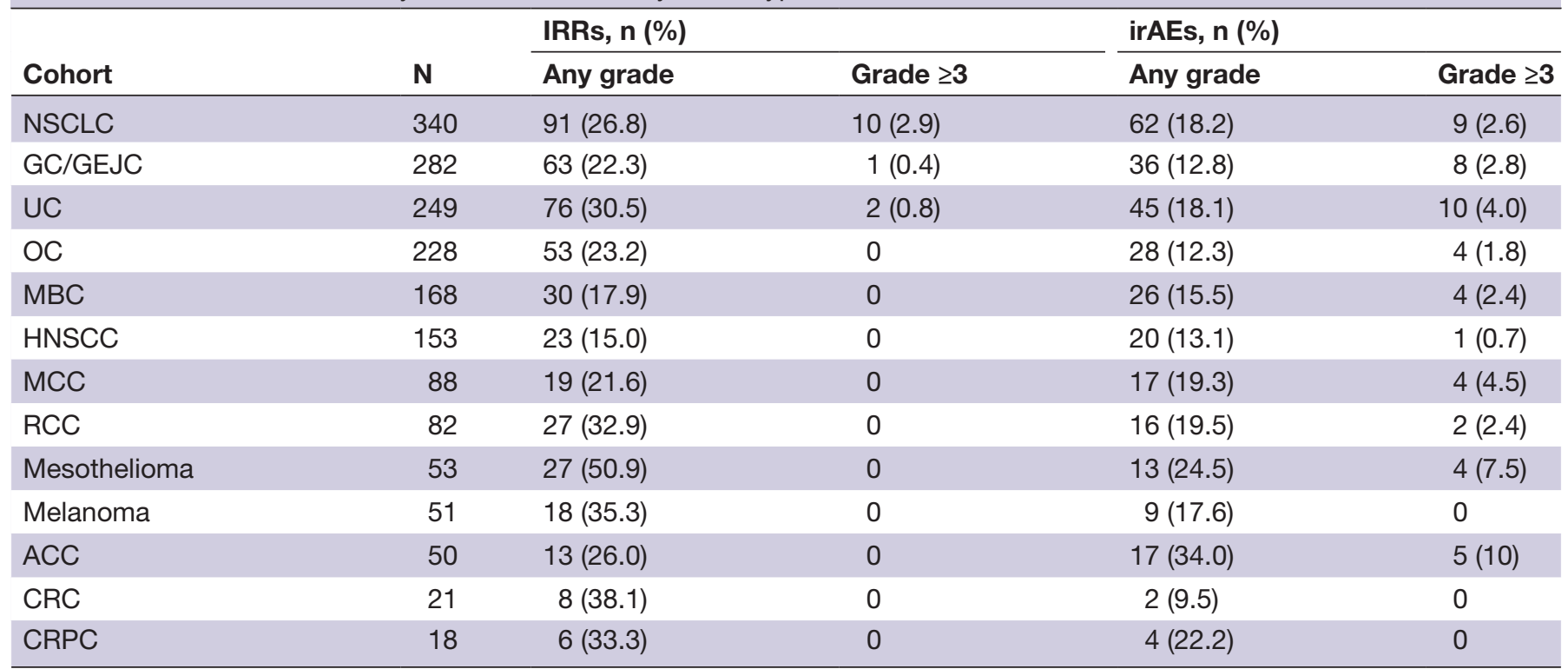

ACC, adrenocortical carcinoma; CRC, colorectal cancer; CRPC, castration-resistant prostate cancer; GC/GEJC, gastric cancer or gastroesophageal junction cancer; HNSCC, head and neck squamous cell carcinoma; irAE, immune-related adverse event; IRR, infusionrelated reaction; MBC, metastatic breast cancer; MCC, Merkel cell carcinoma; NSCLC, non-small cell lung cancer; OC, ovarian cancer; RCC, renal cell carcinoma; UC, urothelial carcinoma.

excluded). Despite high-dose steroids, the subject's condition deteriorated, with a fatal outcome after 4 days. No biopsy or autopsy was performed. Altogether, 542 irAEs were observed: 177 patients $(60.0 \%)$ experienced 1 irAE, $66(22.4 \%)$ experienced 2 irAEs, $24(8.1 \%)$ experienced 3 irAEs and $28(9.6 \%)$ experienced $\geq 4$ irAEs. The most common irAEs were thyroid disorders $(\mathrm{n}=120(6.7 \%))$ and rash $(\mathrm{n}=116(6.5 \%))$ and 39 of 1783 patients $(2.2 \%)$ discontinued avelumab due to irAEs.

The time-dependent Cox model of OS showed a lower risk of death in patients who experienced any-grade irAEs compared with those who did not (HR 0.71, 95\%

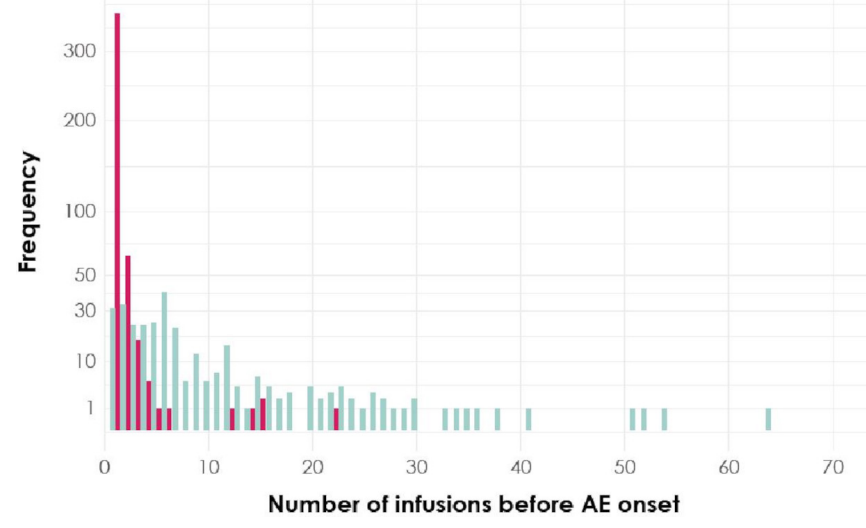

Figure 2 Onset of first AESI. Shown are the number of infusions administered before the onset of the first irAE (aquamarine) or IRR (magenta) across the study population. $A E$, adverse event; AESI, adverse event of special interest; irAE, immune-related adverse event; IRR, infusion-related reaction.
CI 0.59 to 0.85$)$. Supplemental analysis in different irAE subgroups supported this observation of a survival benefit (eg, subsets of patients who experienced grade $\geq 2$ irAEs (HR $0.78,95 \%$ CI 0.63 to 0.95 ), rash irAEs only (HR 0.65 , $95 \%$ CI 0.49 to 0.87 ), thyroid irAEs only (HR $0.53,95 \%$ CI 0.39 to 0.71 ) or subgroups excluding patients receiving steroids (40 mg prednisone or equivalent daily; HR 0.63 , $95 \%$ CI 0.50 to 0.78 ). The multistate model was used to assess the relationship between the likelihood of experiencing a response and the occurrence of an irAE. The estimated transition probabilities of patients progressing from one treatment event to another is shown in figure 3. Each row of graphs in this figure depicts the estimated transition probabilities of the given events over time beginning at three different time points. The model did not suggest that the occurrence of irAEs could predict response (blue arrows and text in figure 1); however, patients with a response were more likely to develop irAEs than those who did not have a response but continued receiving treatment (pink arrows and text in figure 1). At any given point in time, the time-dependent probability for an irAE is higher for a responder than for a non-responder who is receiving treatment. Furthermore, the treatment discontinuation rate did not increase after experiencing an irAE, regardless of whether a response occurred. These results were confirmed in subgroup analyses that accounted for the severity and type of irAEs (results not shown).

Of the 1783 patients in the study, 454 patients $(25.5 \%)$ had IRRs and 13 patients $(0.7 \%)$ had grade $\geq 3$ IRRs. No deaths occurred due to IRRs. Of the subset of patients who experienced IRRs $(n=454), 362$ of these patients $(79.7 \%)$ 

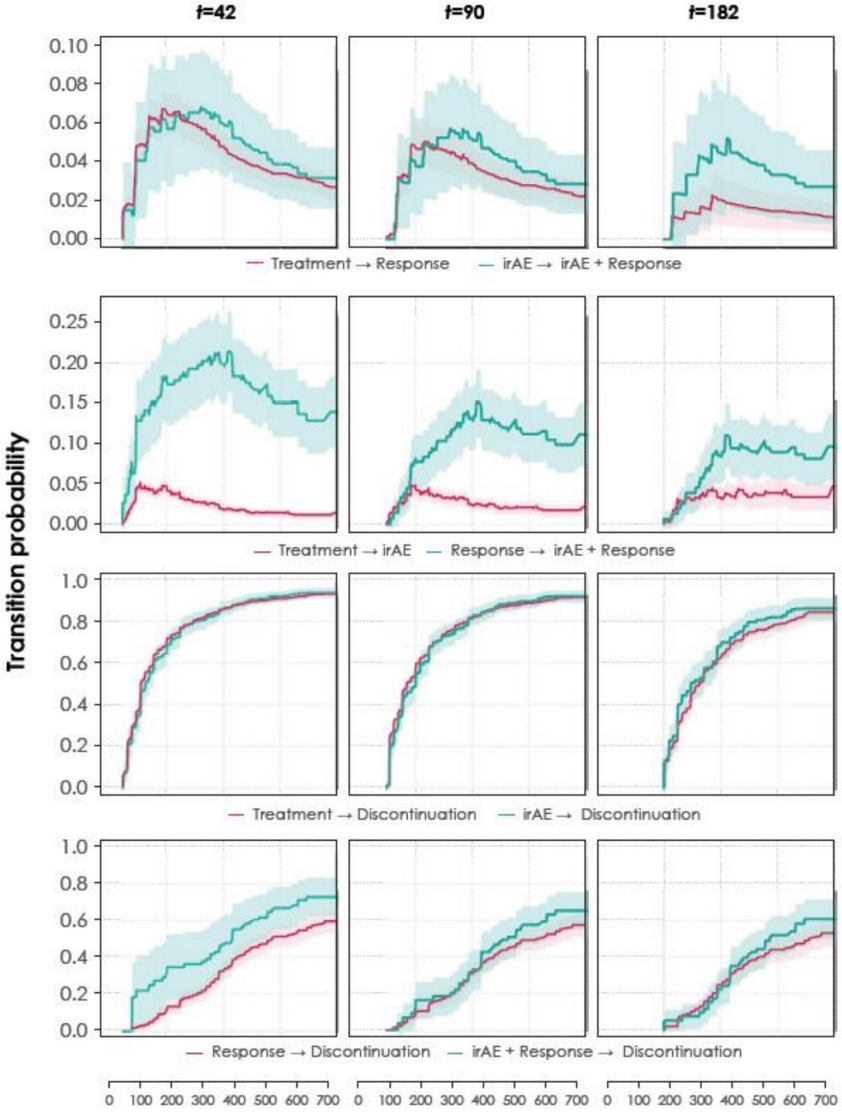

Time, days

Figure 3 Estimated probabilities that a patient transitions between given treatment events. Each row of panels depicts the probability beginning at three different time points (42, 90 and 182 days) after initiation of treatment that a patient transitions from a given model state to another. Shaded areas indicate $90 \%$ Cls. The first row compares the time varying transition probability for response given previous occurrence of irAE or not. The second row compares the time varying transition probability for irAE given previous occurrence of response or not. The third and fourth rows depict time varying transition probability for discontinuation with or without response comparing previous occurrence of irAE or not. irAE, immune-related adverse event.

had a first occurrence of IRRs at the time of first infusion, 63 $(13.9 \%)$ at the second infusion, $17(3.7 \%)$ at the third infusion, $5(1.1 \%)$ at the fourth infusion and $7(1.5 \%)$ at the fifth infusion to the 22nd infusion. A total of $97.4 \%$ of first IRRs occurred before the first response assessment. Of the 454 patients who experienced an IRR, $340(74.9 \%)$ experienced 1 IRR, 88 (19.4\%) experienced 2 IRRs, 20 (4.4\%) experienced 3 IRRs and $6(1.2 \%)$ experienced $\geq 4$ IRRs. Of the 609 total IRR events observed, including recurrences, 287 $(47.1 \%)$ occurred during infusion, $314(51.6 \%)$ occurred later on the day of infusion and $8(1.3 \%)$ occurred on the day after infusion. A total of 30 of 1783 patients (1.7\%) discontinued avelumab due to IRRs.

No association was observed between response and IRRs. IRRs were observed in 57 of 218 patients (26.1\%) who had a response and in 397 of 1565 patients (25.4\%) who did not ( $\chi^{2}$ test $\mathrm{p}=0.87$, with Yates correction). Results were confirmed in relevant analyses of subgroups defined by tumor type from JAVELIN Solid Tumor (eg, UC, melanoma, non-small cell lung cancer (NSCLC), given sufficient sample size) and when limited to clinically relevant IRRs only (grades 2-4). There was a wider distribution of time to response for patients who experienced IRRs, but no conclusions can be drawn due to limited sample size. In addition, DOR did not appear to be associated with the occurrence of IRRs (figure 4). Since a total of $97.4 \%$ of first IRRs occurred before the first response assessment, the analysis would not be needed to account for time dependency. Please note that in the avelumab product label, it is recommended to premedicate with antihistamine and acetaminophen prior to the first four infusions to manage proactively the possibility of IRRs. ${ }^{38} 39$

\section{DISCUSSION}

In this study, we investigated the association between avelumab efficacy and the occurrence of AESIs in patients with a wide variety of solid tumors. Our study addressed the methodological shortcomings present in prior studies using approaches that overcome statistical challenges such as time dependency and event order, thus helping to reconcile contradictory results in the literature.

According to the time-dependent Cox model, patients who experienced irAEs had an improved chance of survival compared with those who did not. Also, patients with irAEs who were not treated with steroids $(40 \mathrm{mg}$ prednisone or equivalent daily) also showed improved chances of survival. One limitation of this analysis is that treatment-related irAEs were only considered within a 30-day window (irAEs can occur months after the last dose of immunotherapy). Although the analysis is still valid, estimates of the true effect of irAE occurrence can be expected to be more pronounced. The multistate model gives meaningful insights to the mechanisms of action in immunotherapy, specifically allowing a flexible event order of response, irAEs and discontinuation. The model did not support the possibility that experiencing irAEs could predict response; not having irAEs did not preclude a response to avelumab. The model also adjusted for treatment duration, as patients with $\mathrm{PR} / \mathrm{CR}$ continued receiving treatment for much longer than patients with SD (median, 14.3 vs 4.6 months). The ideal length of treatment for patients who achieve a PR or CR remains an area of active investigation. However, the suggestion that at each time point the likelihood to develop an irAE was higher for responders emphasizes the need for increased vigilance for irAEs following a response. However, patients who undergo treatment with anti-PD-(L) 1 therapy for extended lengths of time also do not experience an increased cumulative incidence of irAEs. ${ }^{40}$ The probability of treatment discontinuation does not increase with irAEs, which indicates that irAEs are manageable.

In our study, an association between response and IRRs was not observed; the chance of having a response and 


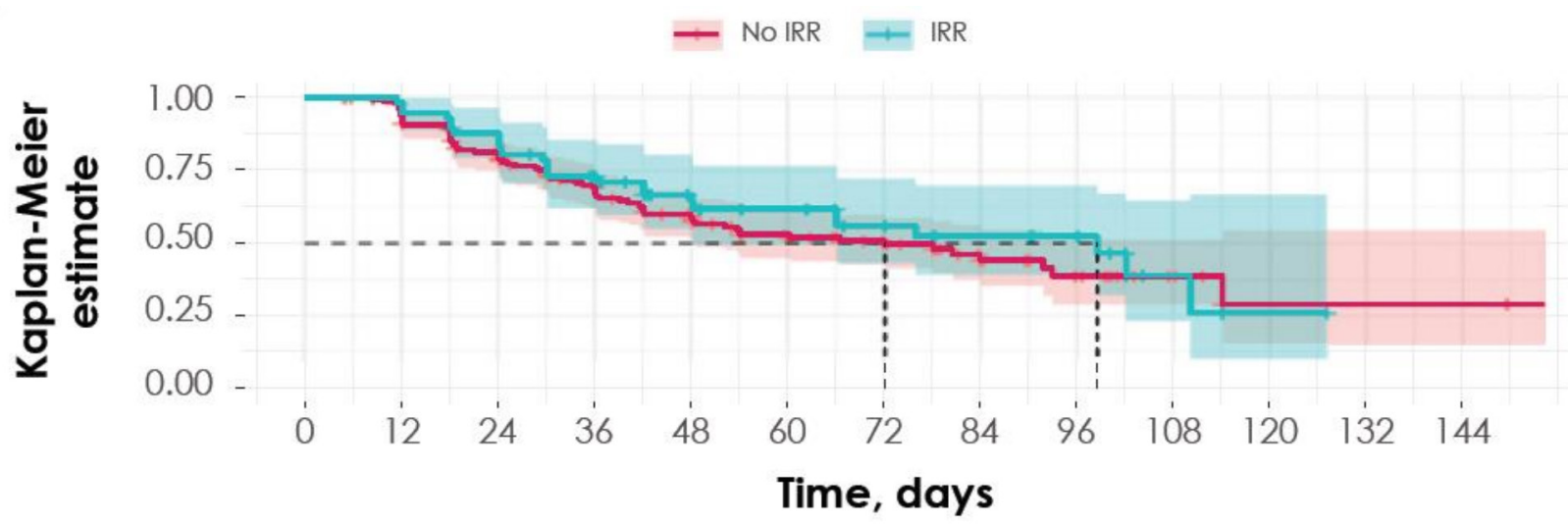

Number at risk (number of events)

\begin{tabular}{r|c|c|c|c|c|c|c|c|c|c|c|c|c|} 
No IRR & $161(0)$ & $150(10)$ & $120(30)$ & $92(46)$ & $71(59)$ & $54(66)$ & $44(68)$ & $23(72)$ & $13(74)$ & $6(74)$ & $2(75)$ & $2(75)$ & $2(75)$ \\
\hline IRR & $57(0)$ & $56(1)$ & $48(7)$ & $37(15)$ & $27(18)$ & $23(20)$ & $18(22)$ & $12(23)$ & $10(23)$ & $3(25)$ & $1(26)$ & $0(26)$ & $0(26)$
\end{tabular}

Number censored

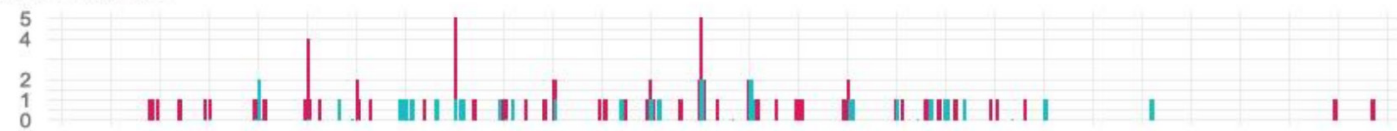

Figure 4 DOR according to occurrence of IRRs. Kaplan-Meier plot shows the estimated DOR based on whether or not a patient experienced an IRR throughout the course of treatment, along with number of patients at risk and breakdown of censored patients at each time point below. Shaded areas indicate 95\% Cls. DOR, duration of response; IRR, infusion-related reaction.

the DOR were not impaired by the occurrence of IRRs. To our knowledge, this is the first study to investigate the association between the occurrence of IRRs and efficacy; although IRRs are AESIs that accompany the use of immunotherapy, their appearance should not be considered an indicator of potential clinical benefit. Thus, the chance of having a response and DOR are not impacted by IRR occurrence.

Studies investigating the association of safety and efficacy in patients receiving $\mathrm{PD}-(\mathrm{L}) 1$ therapy have concluded that the incidence of irAEs is linked to increased OS, progression-free survival (PFS) and/or improved objective response. The occurrence of irAEs has been associated with clinical benefit in the treatment of a wide range of malignancies with various anti-PD-(L) 1 agents. $^{13} 151626282941-46$ However, conflicting results have also been reported in studies on anti-PD-(L) 1 therapy in patients with a variety of tumors, for which there was no significant association between irAEs and OS or PFS. ${ }^{29} 3047$ One reason for the conflicting results may be the methodological limitations in many of the published studies; indeed, many existing analyses are simple association analyses between the occurrence of an irAE and response, which are susceptible to spurious correlation fallacies (ie, that longer treatment is likely to be associated with higher chances of an irAE occurring, as well as the possibility of experiencing a response), and do not adjust for immortal time bias. ${ }^{16} 26-2841444548$ Several studies used landmark analyses to explore the correlation between the incidence of irAEs and efficacy, but predetermined selection of the landmark time points will neglect the value of late-occurring irAEs and their contributions to a potential associative effect. ${ }^{15} 294749$ Our analysis of the timing of AEs during treatment and their relationship with OS was captured using a time-dependent Cox model,${ }^{13}{ }^{14}$ which allows irAEs to occur at any time. Furthermore, method selection often also implies directionality in the association, which can result in incorrect conclusions regarding causality; indeed, some analyses have suggested that irAEs may predict response while not considering flexible event order (ie, that a response or disease progression can occur either before or after an irAE) ${ }^{151628} 2949$ Maher et $a l^{46}$ attempted to consider the possibility that AEs may occur both before and after disease response by fitting two independent models to describe the complex relationship. Our multistate model analysis allows a flexible order of events in the same model while appropriately accounting for treatment discontinuation. Lastly, the sample size used by several studies was very small, thus potentially limiting the conclusions that can be drawn from the analysis. ${ }^{28-30} 43$

In this study, we addressed these limitations by properly accounting for each issue using appropriate methodology. The multistate model allowed us to gain new insights regarding the underlying mechanisms of timings of irAE occurrence, response and discontinuation while allowing flexible event order. Furthermore, the conclusions are based on, to our knowledge, one of the few studies with a large patient population used for the analysis of the association between irAEs and efficacy. Understanding the association between AESIs and response is essential for improving patient care. In conclusion, our study indicates that patients who developed irAEs had an improved chance of survival. Although irAEs could not 
be used to predict a response, patients who responded to avelumab and remained on treatment should be continuously monitored for the emergence of irAEs. However, recent 5-year OS data from patients with advanced NSCLC treated with pembrolizumab show little evidence of late-onset or new toxicities. ${ }^{50}$ This study may also guide future anti-PD $(\mathrm{L}) 1$ treatment discontinuation studies, and future analyses involving other anti- $\mathrm{PD}(\mathrm{L}) 1$ regimens should be conducted with the methodologies outlined here to determine the association between AESIs and efficacy in other treatment contexts.

\section{Author affiliations}

${ }^{1}$ Department of Internal Medicine, University of California Davis Comprehensive Cancer Center, Sacramento, California, USA

${ }^{2}$ EMD Serono Research \& Development Institute, Inc, Billerica, Massachusetts, USA; an affiliate of Merck KGaA, Darmstadt, Germany

${ }^{3}$ Sarah Cannon Research Institute, Florida Cancer Specialists, Sarasota, Florida, USA ${ }^{4}$ Memorial Sloan Kettering Cancer Center, New York, New York, USA

${ }^{5}$ Weill Cornell Medical College, New York, New York, USA

${ }^{6}$ Genitourinary Malignancies Branch, Center for Cancer Research, National Cancer Institute, Bethesda, Maryland, USA

${ }^{7}$ Thoracic and Gastrointestinal Malignancies Branch, Center for Cancer Research, National Cancer Institute, Bethesda, Maryland, USA

${ }^{8}$ EMD Serono, Inc, Rockland, Massachusetts, USA; an affiliate of Merck KGaA

Darmstadt, Germany

${ }^{9}$ Merck KGaA, Darmstadt, Germany

Twitter Andrea B Apolo @apolo_andrea and James L Gulley @gulleyj1

Acknowledgements The authors thank the patients and their families, investigators, coinvestigators, and study teams at each of the participating centers and at Merck KGaA, Darmstadt, Germany, and EMD Serono Research \& Development Institute, Inc., Billerica, MA, USA; an affiliate of Merck KGaA, Darmstadt, Germany. The authors also thank Anja-Helena Loos, Arthur Allignol, Isabella Zwiener, and Anja von Heydebreck for their valuable methodological input.

Contributors Conception and design: JLG, JM, JW, KK and VK. Provision of study materials or patients: ABA, AR, JLG, KK, MRP and SD. Collection and assembly of data: all authors. Data analysis and interpretation: all authors. Manuscript writing: all authors. Final approval of manuscript: all authors.

Funding This work was funded by Merck KGaA, Darmstadt, Germany, as part of an alliance between Merck KGaA, Darmstadt, Germany, and Pfizer, New York, New York, USA. Medical writing support was provided by Irene Minkina, PhD, of ClinicalThinking and funded by Merck KGaA and Pfizer Inc.

Competing interests KK has received research grants from EMD Serono (an affiliate of Merck KGaA, Darmstadt, Germany) and Merck \& Co; has served on advisory boards for EMD Serono and Merck \& $\mathrm{Co}$; and has received an honorarium from Merck \& Co. JM, MB and JW are employees of EMD Serono Research \& Development Institute (an affiliate of Merck KGaA, Darmstadt, Germany). VK was an employee of EMD Serono (an affiliate of Merck KGaA, Darmstadt, Germany) at the time this analysis was conducted. IS is an employee of Merck KGaA, Darmstadt, Germany. SPD reports serving as a consultant or advisor for Amgen, EMD Serono (an affiliate of Merck KGaA, Darmstadt, Germany), GlaxoSmithKline, Immunocore, Immune Design, Incyte, Merck \& Co, and Nektar; has received research grants from Amgen, Bristol Myers Squibb, Deciphera, EMD Serono, Incyte, Merck \& Co, and Nektar; and has received reimbursement for travel and accommodation expenses from Adaptimmune, EMD Serono, Immunocore and Nektar. AR and JLG are employees of the National Cancer Institute, which has a cooperative research and development agreement with EMD Serono (an affiliate of Merck KGaA, Darmstadt, Germany).

\section{Patient consent for publication Not required.}

Ethics approval The trials were conducted in accordance with the ethics principles of the Declaration of Helsinki and the International Council for Harmonisation Guidelines on Good Clinical Practice. The protocols were approved by the institutional review board or independent ethics committee of each center.

Provenance and peer review Not commissioned; externally peer reviewed.
Data availability statement Data are available on reasonable request. For all new products or new indications approved in both the European Union and the USA after January 1 2014, Merck KGaA, Darmstadt, Germany, will share patient-level and study-level data after deidentification, as well as redacted study protocols and clinical study reports from clinical trials in patients. These data will be shared with qualified scientific and medical researchers, on researchers' request, as necessary for conducting legitimate research. Such requests must be submitted in writing to the company's data sharing portal. More information can be found at https:// www.merckgroup.com/en/research/our-approach-to-research-anddevelopment/ healthcare/clinical-trials/commitment-responsible-data-sharing.html. Where Merck $\mathrm{KGaA}$ has a coresearch, codevelopment, or comarketing/copromotion agreement or where the product has been outlicensed, it is recognized that the responsibility for disclosure may be dependent on the agreement between parties. Under these circumstances, Merck KGaA will endeavor to gain agreement to share data in response to requests.

Open access This is an open access article distributed in accordance with the Creative Commons Attribution Non Commercial (CC BY-NC 4.0) license, which permits others to distribute, remix, adapt, build upon this work non-commercially, and license their derivative works on different terms, provided the original work is properly cited, appropriate credit is given, any changes made indicated, and the use is non-commercial. See http://creativecommons.org/licenses/by-nc/4.0/.

Author note Vijay Kasturi: Affiliation at the time the analysis was conducted

\section{ORCID iDs}

Karen Kelly http://orcid.org/0000-0002-2235-6636

Sandra P D'Angelo http://orcid.org/0000-0002-3736-3783

Andrea B Apolo http://orcid.org/0000-0001-9409-1836

Arun Rajan http://orcid.org/0000-0003-2776-3385

James L Gulley http://orcid.org/0000-0002-6569-2912

\section{REFERENCES}

1 Hoos A. Development of immuno-oncology drugs - from CTLA4 to PD1 to the next generations. Nat Rev Drug Discov 2016;15:235-47.

2 La-Beck NM, Jean GW, Huynh C, et al. Immune checkpoint inhibitors: new insights and current place in cancer therapy. Pharmacotherapy 2015;35:963-76.

3 Friedman CF, Proverbs-Singh TA, Postow MA. Treatment of the immune-related adverse effects of immune checkpoint inhibitors: a review. JAMA Oncol 2016;2:1346-53.

4 Vogel WH. Infusion reactions: diagnosis, assessment, and management. Clin J Oncol Nurs 2010;14:E10-21.

5 Thompson JA, Schneider BJ, Brahmer J, et al. Management of immunotherapy-related toxicities, version 1.2019. J Natl Compr Canc Netw 2019;17:255-89.

6 Fucà G, Galli G, Poggi M, et al. Modulation of peripheral blood immune cells by early use of steroids and its association with clinical outcomes in patients with metastatic non-small cell lung cancer treated with immune checkpoint inhibitors. ESMO Open 2019;4:e000457.

7 Horvat TZ, Adel NG, Dang T-O, et al. Immune-related adverse events, need for systemic immunosuppression, and effects on survival and time to treatment failure in patients with melanoma treated with ipilimumab at Memorial Sloan Kettering cancer center. J Clin Oncol 2015;33:3193-8.

8 Michot JM, Bigenwald C, Champiat S, et al. Immune-related adverse events with immune checkpoint blockade: a comprehensive review. Eur J Cancer 2016;54:139-48.

9 Du X, Liu M, Su J, et al. Uncoupling therapeutic from immunotherapy-related adverse effects for safer and effective anti-CTLA-4 antibodies in CTLA4 humanized mice. Cell Res 2018;28:433-47.

10 Hua C, Boussemart L, Mateus C, et al. Association of vitiligo with tumor response in patients with metastatic melanoma treated with pembrolizumab. JAMA Dermatol 2016;152:45-51.

11 Das S, Johnson DB. Immune-related adverse events and anti-tumor efficacy of immune checkpoint inhibitors. J Immunother Cancer 2019;7:306.

12 Ksienski D, Wai ES, Croteau N, et al. Efficacy of nivolumab and pembrolizumab in patients with advanced non-small-cell lung cancer needing treatment interruption because of adverse events: a retrospective multicenter analysis. Clin Lung Cancer 2019;20:e97-106.

13 von Pawel J, Syrigos K, Mazieres J, et al. 1314PAssociation between immune-related adverse events (irAEs) and atezolizumab efficacy in 
advanced NSCLC: analyses from the phase III study OAK. Ann Oncol 2017;28(suppl_5).

14 Freeman-Keller M, Kim Y, Cronin H, et al. Nivolumab in resected and unresectable metastatic melanoma: characteristics of immunerelated adverse events and association with outcomes. Clin Cancer Res 2016;22:886-94.

15 Haratani K, Hayashi H, Chiba Y, et al. Association of immune-related adverse events with nivolumab efficacy in non-small-cell lung cancer. JAMA Oncol 2018;4:374-8.

16 Toi Y, Sugawara S, Kawashima Y, et al. Association of immunerelated adverse events with clinical benefit in patients with advanced non-small-cell lung cancer treated with nivolumab. Oncologist 2018;23:1358-65.

17 Eggermont AMM, Kicinski M, Blank CU, et al. Association between immune-related adverse events and recurrence-free survival among patients with stage III melanoma randomized to receive pembrolizumab or placebo: a secondary analysis of a randomized clinical trial. JAMA Oncol 2020;6:519-27.

18 Das S, Ciombor KK, Haraldsdottir S, et al. Immune-Related adverse events and immune checkpoint inhibitor efficacy in patients with gastrointestinal cancer with Food and Drug Administration-approved indications for immunotherapy. Oncologist 2020. doi:10.1634/ theoncologist.2019-0637. [Epub ahead of print: 14 Jan 2020].

19 Masuda K, Shoji H, Nagashima K, et al. Correlation between immune-related adverse events and prognosis in patients with gastric cancer treated with nivolumab. BMC Cancer 2019;19:974.

20 Teulings H-E, Limpens J, Jansen SN, et al. Vitiligo-like depigmentation in patients with stage III-IV melanoma receiving immunotherapy and its association with survival: a systematic review and meta-analysis. $J$ Clin Oncol 2015;33:773-81.

21 Berner F, Bomze D, Diem S, et al. Association of checkpoint inhibitorinduced toxic effects with shared cancer and tissue antigens in nonsmall cell lung cancer. JAMA Oncol 2019;5:1043-7.

22 Maione P, Rossi A, Di Maio M, et al. Tumor-related leucocytosis and chemotherapy-induced neutropenia: linked or independent prognostic factors for advanced non-small cell lung cancer? Lung Cancer 2009;66:8-14.

23 Soveri LM, Hermunen K, de Gramont A, et al. Association of adverse events and survival in colorectal cancer patients treated with adjuvant 5-fluorouracil and leucovorin: is efficacy an impact of toxicity? Eur J Cancer 2014;50:2966-74.

24 Pérez-Soler R, Chachoua A, Hammond LA, et al. Determinants of tumor response and survival with erlotinib in patients with non-small-cell lung cancer. J Clin Oncol 2004;22:3238-47.

25 Sung MW, Finn RS, Qin S, et al. Association between overall survival and adverse events with lenvatinib treatment in patients with hepatocellular carcinoma (reflect). J Clin Oncol 2019;37:317.

26 Grangeon M, Tomasini P, Chaleat S, et al. Association between immune-related adverse events and efficacy of immune checkpoint inhibitors in non-small-cell lung cancer. Clin Lung Cancer 2019;20:201-7.

27 Judd J, Zibelman M, Handorf E, et al. Immune-related adverse events as a biomarker in non-melanoma patients treated with programmed cell death 1 inhibitors. Oncologist 2017;22:1232-7.

28 Teraoka S, Fujimoto D, Morimoto T, et al. Early immune-related adverse events and association with outcome in advanced non-small cell lung cancer patients treated with nivolumab: a prospective cohort study. J Thorac Oncol 2017;12:1798-805.

29 Sato K, Akamatsu H, Murakami E, et al. Correlation between immune-related adverse events and efficacy in non-small cell lung cancer treated with nivolumab. Lung Cancer 2018:115:71-4.

30 Nakamura Y, Tanaka R, Asami Y, et al. Correlation between vitiligo occurrence and clinical benefit in advanced melanoma patients treated with nivolumab: a multi-institutional retrospective study. $J$ Dermatol 2017;44:117-22.

31 Heery CR, O'Sullivan-Coyne G, Madan RA, et al. Avelumab for metastatic or locally advanced previously treated solid tumours (javelin solid tumor): a phase $1 \mathrm{~A}$, multicohort, dose-escalation trial. Lancet Oncol 2017;18:587-98.
32 Chen X, Song X, Li K, et al. FcyR-Binding is an important functional attribute for immune checkpoint antibodies in cancer immunotherapy. Front Immunol 2019;10:292.

33 Boyerinas B, Jochems C, Fantini M, et al. Antibody-dependent cellular cytotoxicity activity of a novel anti-PD-L1 antibody avelumab (MSB0010718C) on human tumor cells. Cancer Immunol Res 2015;3:1148-57.

34 Kelly K, Infante JR, Taylor MH, et al. Safety profile of avelumab in patients with advanced solid tumors: a pooled analysis of data from the phase 1 JAVELIN solid tumor and phase 2 JAVELIN Merkel 200 clinical trials. Cancer 2018;124:2010-7.

35 Beyersmann J, Allignol A, Schumacher M. Competing risks and multistate models with R. Springer, 2012.

36 de Wreede LC, Fiocco M, Putter H. The mstate package for estimation and prediction in non- and semi-parametric multi-state and competing risks models. Comput Methods Programs Biomed 2010;99:261-74.

37 LCd W, Fiocco M, Putter H. mstate: an R package for the analysis of competing risks and multi-state models. J Stat Softw 2011;38:1-30.

38 Bavencio (avelumab) Prescribing information. EMD Serono, Inc; an affiliate of Merck KGaA, Darmstadt, Germany, 2020. Available: https://www.emdserono.com/us-en/pi/bavencio-pi.pdf [Accessed 12 Oct 2020].

39 Bavencio (avelumab) Summary of product characteristics. Merck KGaA, Darmstadt, Germany, 2020. Available: https://www.medicines. org.uk/emc/product/8453/smpc\#gref [Accessed 12 Oct 2020].

40 Postow MA, Sidlow R, Hellmann MD. Immune-related adverse events associated with immune checkpoint blockade. N Engl J Med 2018;378:158-68.

41 Moor RJ, Roberts KE, Mason R, et al. Immune-related adverse events and nivolumab outcomes in non-small cell lung cancer patients: a multi-institutional, retrospective cohort study. J Clin Oncol 2018;36(15_suppl):9067.

42 Vitale MG, Pipitone S, Scagliarini S, et al. 891PCorrelation between immuno-related adverse events (IRAEs) occurrence and clinical outcome in metastatic renal cell carcinoma (mRCC) patients treated with nivolumab: IRAENE trial, an Italian multi-institutional retrospective study. Ann Oncol 2018;29(suppl_8).

43 Okada N, Kawazoe H, Takechi K, et al. Association between immune-related adverse events and clinical efficacy in patients with melanoma treated with nivolumab: a multicenter retrospective study. Clin Ther 2019;41:59-67.

44 Quach HT, Dewan AK, Davis EJ, et al. Association of antiprogrammed cell death 1 cutaneous toxic effects with outcomes in patients with advanced melanoma. JAMA Oncol 2019;5:906-8.

45 Rogado J, Sánchez-Torres JM, Romero-Laorden N, et al. Immunerelated adverse events predict the therapeutic efficacy of anti-PD-1 antibodies in cancer patients. Eur J Cancer 2019;109:21-7.

46 Maher VE, Fernandes LL, Weinstock C, et al. Analysis of the association between adverse events and outcome in patients receiving a programmed death protein 1 or programmed death ligand 1 antibody. J Clin Oncol 2019;37:2730-7.

47 Kfoury M, Voisin A-L, Najean M, et al. 1141PDAssociation between immune-related adverse events and efficacy in patients treated with anti-PD-(L)1. Ann Oncol 2018;29(suppl_8).

48 Abu-Sbeih H, Tang T, Ali FS, et al. The impact of immune checkpoint inhibitor-related adverse events and their immunosuppressive treatment on patients' outcomes. J Immunother Precis Oncol 2018;1:7.

49 Ricciuti B, Genova C, De Giglio A, et al. Impact of immune-related adverse events on survival in patients with advanced non-small cell lung cancer treated with nivolumab: long-term outcomes from a multi-institutional analysis. J Cancer Res Clin Oncol 2019;145:479-85.

50 Garon EB, Hellmann MD, Rizvi NA, et al. Five-Year overall survival for patients with advanced non-small-cell lung cancer treated with pembrolizumab: results from the phase I KEYNOTE-001 study. J Clin Oncol 2019;37:JCO1900934:2518-27. 